\title{
A special issue on active disturbance rejection control (ADRC)
}

\author{
Zhiqiang Gao' ${ }^{1}$ Yi Huang ${ }^{2,3}$
}

Received: 5 February 2021 / Accepted: 6 February 2021 / Published online: 21 April 2021

(c) South China University of Technology, Academy of Mathematics and Systems Science, CAS and Springer-Verlag GmbH Germany, part of Springer Nature 2021

Key to any control system is a mechanism that regulates certain attributes of a dynamic system, engineered or natural. In this field of profound impacts to all branches of engineering, know-how is distinguished from know-why, practice from understanding, and experience from knowledge. The practice of automatic control in the modern era began with the eighteenth century flyball governor, the predecessor of today's industry-dominant PID technology, and its theory with the 1868 paper of James Clerk Maxwell, titled simply "On Governors".

If the maturity of a field of engineering is to be measured by the gap between theory and practice, control engineering is still quite young. The dominance of PID in practice, in contrast to the exponential growth in mathematical control theory since WWII, has puzzled generations of researchers and practitioners. But control is not dead, as proclaimed by some in the past. How can such a thriving field of engineering practice be dead? It's the science behind it that needs self-correction, and a new paradigm if you will. In comes ADRC, the subject of this special issue.

If "everything must justify its existence before the judgment-seat of reason or give up existence,"1 so does ADRC. And such justification is to be found in the relevance to engineering practice, we believe. Practice, after all, is the sole criterion for the truth. This recognition was a watershed moment in 1978; so is it for the reckoning in control theory.

This issue begins with "On transitioning from PID to ADRC in thermal power plants" by Wu et al. from Tsinghua University, a survey on the paradigm-shift in the baseline

\footnotetext{
Zhiqiang Gao

z.gao@csuohio.edu
}

1 Center for Advanced Control Technologies, Cleveland State University, Cleveland, Ohio, USA

2 Key Laboratory of Systems and Control, Academy of Mathematics and Systems Science, Chinese Academy of Sciences, Beijing 100190, China

3 School of Mathematical Sciences, University of Chinese Academy of Sciences, Beijing 100049, China technology in coal-fired powerplants. In support of such adoptions in industry, Herbst from the University of Applied Sciences Zwickau, Germany, explores the important issues such as compatibility, tuning, and computational efficiency, etc.

The willingness by ADRC researchers to confront bottleneck in industry practice is key to the long-term success, so is the willingness to shape-shift the ADRC design for a class of problems that are otherwise not covered in its original formulation. This is shown by Zhong et al. in tackling the problem of flexible aircraft, where the controlled variable tends to vibrate and is not measurable, and by Guo et al. in embedding an ESO in the traditional PLL to counteract disturbances faced by the grid-connected converters. Mohorcic and Dong similarly embedded an ESO in the pressure loop for a pneumatic servo system, as do Rojas-Cubides et al. and Madonski et al. in two case studies of DC-DC buck converters, respectively.

Time and again these studies show that in so called "applications", what to be treasured is not a rigid adoption of a set of ADRC equations, but the insightful recognition of the essence of the problem at hand and the creative use of the basic principles. Once the problem is properly diagnosed and formulated, the solution is almost obvious.

No special issue is complete without putting ADRC in the context of the existing framework of various branches of control theory, from which a contrast or connection can be made. To this end, Sira-Ramirez et al. from Mexico continued the work in exhibiting connections among the GPI, the classical compensation, and the ADRC (with reduced order ESO) under certain conditions, this time by way of an EIEF approach for a class of nonlinear SISO Lagrangian systems; Otero-Leal et al. from Colombia provided a unified ADRC solution for fractional- and integer order plant with a flat output; Zhang et al, proposed an extended state-based Kalman filter for a class of nonlinear time-varying stochastic systems with uncertain dynamics and measurement bias.

\footnotetext{
${ }^{1}$ Frederich Engels (1878). Herr Eugen Dühring's Revolution in Science.
} 
2021 marks the twentieth anniversary of ADRC as it was presented comprehensively for the first time in the English literature in the 2001 IEEE Conference on Decision and Control. With groundbreaking industry adoptions and the supporting scholarly work far too wide to be captured here, we hope the readers come to see, nonetheless, how the theory-practice gap is narrowed via the bridge called ADRC, one step at a time. We look forward to the future of control engineering with anticipation, a future of unity in knowing and doing.

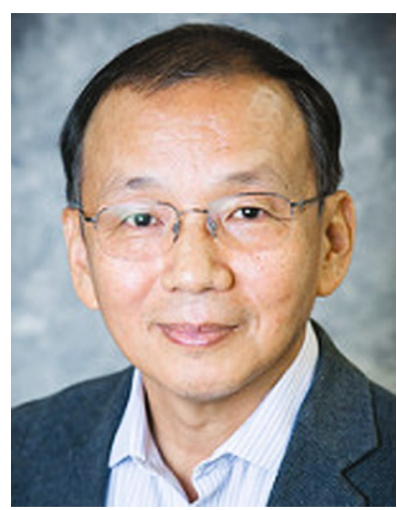

Zhiqiang Gao received his Ph.D. in Electrical Engineering from the University of Notre Dame in 1990 and has taught at Cleveland State University ever since. Collaborating with Prof. Jingqing Han, Dr. Gao worked quietly on active disturbance rejection control for over 20 years, nurturing it from its early, conceptual stage to a maturing and emerging industrial control technology. Asking basic, rudimentary question in research and in teaching,
Dr. Gao and his team find creative solutions in practice and vitality in education.

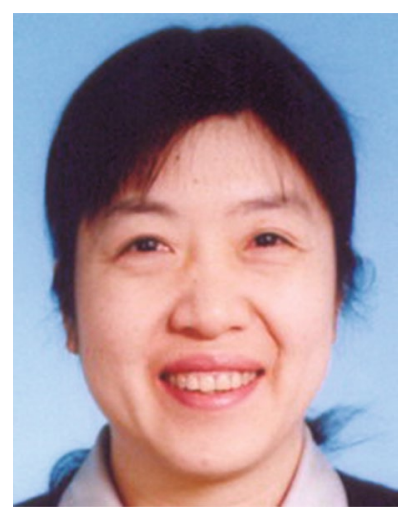

Yi Huang received the Ph.D. degree in Control Theory and Its Applications from Southeast University, Nanjing, in 1995. From 1995 to 1997 , she worked as a postdoctoral fellow in the Institute of Systems Science, Chinese Academy of Sciences. From 1997, she has been at the Key Laboratory Systems and Control, Chinese Academy of Sciences as an assistant professor, and then associate professor, and professor. Her research work is concentrated on the estimation and control of nonlinear uncertain systems, particularly in applications and analysis of the active disturbance rejection control. 